\title{
Inapproximability results for orthogonal rectangle packing problems with rotations
}

\author{
Miroslav Chlebík ${ }^{1}$ and Janka Chlebíková ${ }^{2 \star}$ \\ 1 MPI for Mathematics in the Sciences, D-04103 Leipzig, Germany, \\ chlebik@mis.mpg.de \\ 2 Faculty of Mathematics, Physics and Informatics, Mlynská dolina, 84248 \\ Bratislava, Slovakia, chlebikova@fmph.uniba.sk
}

\begin{abstract}
Recently Bansal and Sviridenko [4] proved that there is no asymptotic PTAS for 2-Dimensional Orthogonal Rectangle Bin PACKING without rotations allowed, unless $\mathrm{P}=\mathrm{NP}$. We show that similar approximation hardness results hold for several rectangle packing problems even if rotations by ninety degrees around the axes are allowed. Moreover, for some of these problems we provide explicit lower bounds on asymptotic approximation ratio of any polynomial time approximation algorithm.
\end{abstract}

\section{Introduction}

We focus on orthogonal packing problems of rectangles into bins in 2 and 3dimensions, where ninety-degree rotations of rectangles around any of the axes are allowed. These problems have many real-world applications in areas like job scheduling, container loading, and cutting objects out of a strip of material in such a way that the amount of material wasted is minimal.

Notation and terminology. Throughout this paper we only consider offline versions of the problems. In all 2-dimensional variants of the problems, the input consists of a list $\mathscr{L}=\left\{R^{1}, R^{2}, \ldots, R^{n}\right\}$ of 2 -dimensional rectangles in the Euclidean space $\mathbb{R}^{2}$ and a 2-dimensional rectangular bin $\mathbb{B}=\left[0, b_{1}\right] \times\left[0, b_{2}\right]$ (for which the notation $\left(b_{1}, b_{2}\right)$ is used as well). Each rectangle $R^{i}$ is given with an (initial) orientation related to the coordinate axes and side-lengths denoted as $\left(w\left(R^{i}\right), h\left(R^{i}\right)\right)$ and called width and height, respectively. The generalization to the higher dimensions is straightforward. In the 3-dimensional strip version of the problems we suppose that the last dimension of the bin $\mathbb{B}$ is unlimited and we call such bin $\mathbb{B}=\left(b_{1}, b_{2}, \infty\right)$ a strip. All rectangles of the list $\mathscr{L}$ need to be packed into bins without overlap. The most interesting and well-studied version of these problems is the so-called orthogonal version, where the edges of packed rectangles and bins are always parallel to the coordinate axes. In problems without rotations rectangles have to be placed into the bin with given orientation and a feasible solution is called oriented packing. In problems with rotations allowed rectangles to be placed may be rotated around any of the axes by $90^{\circ}$

* The author has been supported by VEGA grant no. 1/3106/06. 
and a feasible solution is referred to as $r$-packing. In the 3-dimensional case, if only rotations around the $z$-axis (the last one) are allowed, a packing is called $z$-oriented.

Given a list $\mathscr{L}$ of 2 -dimensional rectangles and a 2 -dimensional bin $\mathbb{B}=$ $\left(b_{1}, b_{2}\right)$. The goal of 2-Dimensional Bin PACKIng (2-BP) and 2-Dimensional Bin Packing With Rotations $\left(2-\mathrm{BP}^{r}\right)$ is to find an oriented packing and an $r$-packing, respectively, of all rectangles of $\mathscr{L}$ into the minimum number of copies of $\mathbb{B}$. In 3 -dimensional strip versions of the problems, a list $\mathscr{L}$ of 3 dimensional rectangles and a 3 -dimensional strip $\mathbb{B}=\left(b_{1}, b_{2}, \infty\right)$ are given. In the problems 3-Dimensional Strip Packing (3-SP) and 3-Dimensional StriP PACKING WITH Rotations $\left(3-\mathrm{SP}^{r}\right)$ we are looking for an oriented packing and an $r$-packing, respectively, that minimizes $h$ such that all rectangles of $\mathscr{L}$ are packed into the bin $\left(b_{1}, b_{2}, h\right)$. If only $90^{\circ}$ rotations around the $z$-axis (the unlimited direction of the strip $\mathbb{B}$ ) are allowed, the problem is called $z$-ORIENTED 3-Dimensional StriP PACKInG.

The standard measure of algorithm quality for bin packing problems is the asymptotic approximation ratio. For a minimization problem it is defined as $\rho_{\mathcal{A}}^{\infty}=\lim _{n \rightarrow \infty} \sup _{I}\left\{\frac{\mathcal{A}(I)}{\mathrm{OPT}(I)}: \mathrm{OPT}(I) \geq n\right\}$, where $I$ ranges over the set of all problem instances, and $\mathcal{A}(I)$ (resp. OPT $(I)$ ) denote the value of the solution returned by $\mathcal{A}$ (resp. the optimum value) for an input instance $I$. For a maximization problem, $\frac{\mathcal{A}(I)}{\mathrm{OPT}(I)}$ is replaced by $\frac{\mathrm{OPT}(I)}{\mathcal{A}(I)}$ so that always $\rho_{\mathcal{A}}^{\infty} \geq 1$. We say, that a problem admits an asymptotic approximation scheme (shortly, APTAS), if for any $\varepsilon>0$ there is a polynomial time algorithm with an asymptotic approximation ratio less than $1+\varepsilon$. For other optimization terminology we refer to Ausiello et al. [1].

Overview. For 1-BP, Fernandez de la Vega \& Lueker [10] designed an APTAS. More precisely, for any positive integer $k$ they provided a polynomial time algorithm $\mathcal{A}_{k}$ that uses at most $\left(1+\frac{1}{k}\right)$ OPT +1 bins. Later, Karmarkar \& Karp [15] gave a single algorithm with asymptotic approximation ratio 1 that uses $\mathrm{OPT}+O\left(1+\log ^{2} \mathrm{OPT}\right)$ bins. For the 2-BP problem Caprara [5] presented an algorithm with currently the best asymptotic approximation ratio 1.691 . On the negative side, Bansal \& Sviridenko [4] proved that there is no APTAS for 2-BP, unless $\mathrm{P}=\mathrm{NP}$. Interestingly, they provided an APTAS for a restricted version of $d$-BP in which the items and the bins are $d$-cubes; this result was independently obtained by Correa \& Kenyon [8]. For 3-BP, Li \& Cheng [17] and Csizik $\&$ van Vliet [9] designed algorithms with asymptotic ratio at most 4.84 . This asymptotic ratio was later improved to $4+\varepsilon$ by Jansen $\&$ Solis-Oba [11]. The algorithms from [17] and [9] generalize to the problem $d$-BP with asymptotic approximation ratio at most $1.691^{d}$. For the problem $2-\mathrm{SP}$, the breakthrough result was obtained by Kenyon \& Rémila [16] who gave an APTAS. For 3-SP, Miyazawa \& Wakabayashi [19] presented an algorithm with asymptotic approximation ratio at most 2.64, which was improved to $2+\varepsilon$ by Jansen \& Solis-Oba [11]. On the other hand, it is easy to see that approximation hardness result for 2-BP implies that no APTAS for 3-SP can exist, unless $\mathrm{P}=\mathrm{NP}$ (see Section 2.1 for more details). 
When ninety-degree rotations are allowed, only weaker results are known. Some algorithms for the versions without rotations provide upper bounds on asymptotic approximation ratio for versions with rotations allowed as well. The results by Miyazawa \& Wakabayashi [18] were the first ones where rotations are exploited in non-trivial way. Currently the best upper bounds on asymptotic approximation ratio for the problems $2-\mathrm{BP}^{r}, 3-\mathrm{BP}^{r}, 3-\mathrm{SP}^{r}$, and $3-\mathrm{SP}^{z}$, are $2+\varepsilon, 4.89,2.76$, and 2.64, respectively, see [19] and [12]. Moreover, Jansen \& Stee provided an APTAS for 2-SP ${ }^{r}$ ([12]).

Rectangle Packing without and with Rotations. When dealing with packing problems without rotation, one can always assume that a bin $\mathbb{B}$ is a unit cube (resp., a base of a strip $\mathbb{B}$ is a unit cube), as the problems are invariant under heterogeneous scaling, i.e., the one which scales by different factors in different coordinate directions. However, this is not true for problems with rotations allowed. It is unclear if the problems with rotations allowed, where the bin $\mathbb{B}$ is a unit cube, are easier to approximate than the general one. For some problems, algorithms with better asymptotic approximation ratio were suggested in such restricted case. For example, when a base of the strip in the problem $3-\mathrm{SP}^{z}$ is a unit square, an algorithm with asymptotic approximation ratio at most 2.528 is known [18].

Using heterogeneous scaling one can show that 2-BP can be viewed as a particular case of general $2-\mathrm{BP}^{r}$ with highly excentric instances. Let a list $\mathscr{L}=$ $\left(R^{1}, R^{2}, \ldots, R^{n}\right)$ of rectangles with dimensions $R^{i}=\left(r_{1}^{i}, r_{2}^{i}\right), i=1,2, \ldots, n$, and a bin $\mathbb{B}=\left(b_{1}, b_{2}\right)$ be an instance of 2-BP. One can find positive scaling factors $\lambda_{1}, \lambda_{2}$, and use scaling $\left(x_{1}, x_{2}\right) \mapsto\left(\lambda_{1} x_{1}, \lambda_{2} x_{2}\right)$ to map any $R^{i}$ to $\widetilde{R}^{i}=\left(\widetilde{r}_{1}^{i}, \widetilde{r}_{2}^{i}\right)$, and the bin $\mathbb{B}$ to $\widetilde{\mathbb{B}}=\left(\widetilde{b}_{1}, \widetilde{b}_{2}\right)$, so that it holds that $\min \left\{\widetilde{r}_{1}^{i}: 1 \leq i \leq n\right\}>\widetilde{b}_{2}$. It is easy to see that the only way a rectangle $\widetilde{R}^{i}$ can fit into the bin $\widetilde{\mathbb{B}}$, even if ninety-degree rotations are allowed, is that $\widetilde{R}^{i}$ is not rotated. Similarly, 3-SP can be handled as a particular case of $3-\mathrm{SP}^{r}$ or $3-\mathrm{SP}^{z}$. Thus, for problems 2$\mathrm{BP}^{r}, 3-\mathrm{SP}^{r}$, and $3-\mathrm{SP}^{z}$ without any restriction on the bin $\mathbb{B}$, non-existence of an APTAS easily follows from results by Bansal \& Sviridenko [4] for 2-BP (see Sections 2 and 2.1 for more details). However, for the most interesting case of a unit square bin $\mathbb{B}$, one can hardly obtain hardness results in such a way.

Main results. In this paper we prove non-existence of an APTAS (unless $\mathrm{P}=$ NP) for 2-Dimensional Bin Packing With Rotations into unit square bins (Section 2), 3-Dimensional Strip PACKIng With Rotations and $z$-ORIEnted 3-Dimensional Strip Packing (Section 2.1) into a strip with unit square base. The methods allow to give explicit lower bounds on asymptotic approximation ratio of any polynomial time approximation algorithm (unless $\mathrm{P}=\mathrm{NP}$ ). For example, we provide a lower bound $1+\frac{1}{3792}$ for 2-Dimensional Bin PACKInG With Rotations, and $1+\frac{1}{2196}$ for the same problem without rotations.

We prove also non-existence of an APTAS for a related 3-dimensional packing problem where the goal is to pack the maximum number of rectangles from a given collection into a single cube bin (Section 3).

General technique. Recall, that for pairwise disjoint sets $X, Y, Z$, and a set of ordered triples $T \subseteq X \times Y \times Z$, a matching in $T$ is a subset $M \subseteq T$ in which 
no two ordered triples in $M$ agree in any coordinate. The goal of the MAXIMUM 3-Dimensional Matching problem (shortly, MAX-3DM) is to find a matching in $T$ of maximum cardinality. A $k$-bounded MAX-3DM is restricted to instances, in which each element of $X \cup Y \cup Z$ occurs at most $k$ times in $T$.

Kann [14] showed that the 3-bounded MAX-3DM problem is Max SNPcomplete (hence also APX-complete). Thus, using PCP-theorem, the existence of a PTAS for it would imply that $\mathrm{P}=\mathrm{NP}$. Petrank [20] proved a refined approximation hardness result that an NP-hard gap occurs also on instances with perfect matching. Unfortunately, the estimates that are implicit in his proof provide lower bound $1+\varepsilon$ with extremely small $\varepsilon>0$. To achieve explicit inapproximability results it is more convenient to use the following NP-hard gap type result for 2-bounded instances of MAX-3DM.

Theorem A. [7] There are instances $T \subseteq X \times Y \times Z$ of 2-bounded MAX$3 \mathrm{DM}$ with $|X|=|Y|=|Z|(:=q)$ and every element of $X \cup Y \cup Z$ occurring in exactly 2 triples in $T$ such that it is $\mathrm{NP}$-hard to distinguish between instances with $\mathrm{OPT}(T)>0.979338843 q$ and $\mathrm{OPT}(T)<0.9690082645 q$.

Both mentioned approximation hardness results for bounded MAX-3DM suit well as a starting point to inapproximability results for various (multidimensional) packing, covering, and scheduling problems, see e.g., [21], [6], and [4].

\section{2-dimensional Bin Packing with Rotations}

In this section we build on ideas from [4] and introduce a general parametrised version of a gap preserving reduction from bounded MAX-3DM to 2-DIMENSIONAL BIN PACKING. We show that with properly chosen parameters this reduction can be used to obtain approximation hardness results for 2-DIMENSIONAL BIN PACKING With Rotations into unit square bin.

The Bin Packing reduction. Let $\mathcal{T}$ be an infinite set of instances (ordered triples) $T$ of MAX-3DM with the optimum value $\mathrm{OPT}(T)$, with the property that for some efficiently computable function $\alpha(T)<\beta(T)$ it is NP-hard to decide of whether $\operatorname{OPT}(T) \geq \beta(T)$, or $\operatorname{OPT}(T)<\alpha(T)$. For a fixed instance $T \in \mathcal{T}$ let $X:=\Pi_{1}(T), Y:=\Pi_{2}(T)$, and $Z:=\Pi_{3}(T)$, where $\Pi_{i}(T)=\left\{p_{i}\right.$ : $\left.\left(p_{1}, p_{2}, p_{3}\right) \in T\right\}$ for $i=1,2,3$, and $X, Y, Z$ are pairwise disjoint sets. The objects in $X, Y, Z$, and $T$ will be denoted as $\left\{x_{i}: 1 \leq i \leq|X|\right\},\left\{y_{j}: 1 \leq j \leq\right.$ $|Y|\},\left\{z_{k}: 1 \leq k \leq|Z|\right\}$, and $\left\{t_{l}: 1 \leq l \leq|T|\right\}$, respectively. (In fact, we will use this general reduction for instances from Theorem A, where $|X|=|Y|=|Z|$ holds.) Of course, any $t_{l} \in T$ is of the form $t_{l}=\left(x_{i}, y_{j}, z_{k}\right) \in X \times Y \times Z$. Let $n=|X|+|Y|+|Z|, q=\max \{|X|,|Y|,|Z|\}$, and $r=32 q$. The reduction has several parameters: a gap location $\beta(T), \delta \in\left(0, \frac{1}{500}\right]$, and $p \in\left[\frac{1}{4}+9 \delta, \frac{1}{2}-20 \delta\right]$.

We first define an integer for each object in $X, Y, Z$, and $T$ as follows: $x_{i}^{\prime}=i r^{3}+i^{2} r+1$, for $1 \leq i \leq|X|, y_{j}^{\prime}=j r^{6}+j^{2} r^{4}+2$, for $1 \leq j \leq|Y|$, $z_{k}^{\prime}=k r^{9}+k^{2} r^{7}+4$, for $1 \leq k \leq|Z|$. For each triple $t_{l}=\left(x_{i}, y_{j}, z_{k}\right) \in T$ we define an integer $t_{l}^{\prime}=r^{10}-x_{i}^{\prime}-y_{j}^{\prime}-z_{k}^{\prime}+15$. Put $c=\frac{r^{10}+15}{\delta}$ and observe 
that $0<x_{i}^{\prime}, y_{j}^{\prime}, z_{k}^{\prime}<\frac{\delta c}{10}$ for all $i, j, k$, and $t_{l}^{\prime}+x_{i}^{\prime}+y_{j}^{\prime}+z_{k}^{\prime}=c \delta$ whenever $t_{l}=\left(x_{i}, y_{j}, z_{k}\right) \in T$.

For each $x_{i} \in X$ (resp., $y_{j} \in Y$ and $z_{k} \in Z$ ) we define a pair of rectangles $A_{X, i}, A_{X, i}^{\prime}$ (resp., $A_{Y, j}, A_{Y, j}^{\prime}$ and $A_{Z, k}, A_{Z, k}^{\prime}$ ) with width about $\frac{1}{4}$ and with heights about $\frac{1}{2}+p$ and $\frac{1}{2}-p$ as follows:

$$
\begin{aligned}
& A_{X, i}=\left(\frac{1}{4}-4 \delta+\frac{x_{i}^{\prime}}{c}, \frac{1}{2}+p+4 \delta-\frac{x_{i}^{\prime}}{c}\right), A_{X, i}^{\prime}=\left(\frac{1}{4}+4 \delta-\frac{x_{i}^{\prime}}{c}, \frac{1}{2}-p-4 \delta+\frac{x_{i}^{\prime}}{c}\right), \\
& A_{Y, j}=\left(\frac{1}{4}-3 \delta+\frac{y_{j}^{\prime}}{c}, \frac{1}{2}+p+3 \delta-\frac{y_{j}^{\prime}}{c}\right), A_{Y, j}^{\prime}=\left(\frac{1}{4}+3 \delta-\frac{y_{j}^{\prime}}{c}, \frac{1}{2}-p-3 \delta+\frac{y_{j}^{\prime}}{c}\right), \\
& A_{Z, k}=\left(\frac{1}{4}-2 \delta+\frac{z_{k}^{\prime}}{c}, \frac{1}{2}+p+2 \delta-\frac{z_{k}^{\prime}}{c}\right), A_{Z, k}^{\prime}=\left(\frac{1}{4}+2 \delta-\frac{z_{k}^{\prime}}{c}, \frac{1}{2}-p-2 \delta+\frac{z_{k}^{\prime}}{c}\right) .
\end{aligned}
$$

For each $t_{l} \in T$ we define two rectangles $B_{l}$ and $B_{l}^{\prime}$ such that

$B_{l}=\left(\frac{1}{4}+8 \delta+\frac{t_{l}^{\prime}}{c}, \frac{1}{2}+p+\delta-\frac{t_{l}^{\prime}}{c}\right) \quad$ and $\quad B_{l}^{\prime}=\left(\frac{1}{4}-8 \delta-\frac{t_{l}^{\prime}}{c}, \frac{1}{2}-p-\delta+\frac{t_{l}^{\prime}}{c}\right)$.

Let $\mathscr{A}_{X}=\left\{A_{X, 1}, A_{X, 2}, \ldots, A_{X,|X|}\right\}, \mathscr{A}_{X}^{\prime}=\left\{A_{X, 1}^{\prime}, A_{X, 2}^{\prime}, \ldots, A_{X,|X|}^{\prime}\right\}$ and define sets of rectangles $\mathscr{A}_{Y}, \mathscr{A}_{Y}^{\prime}, \mathscr{A}_{Z}$, and $\mathscr{A}_{Z}^{\prime}$ analogously. Put $\mathscr{A}=\mathscr{A}_{X} \cup \mathscr{A}_{Y} \cup \mathscr{A}_{Z}$ and $\mathscr{A}^{\prime}=\mathscr{A}_{X}^{\prime} \cup \mathscr{A}_{Y}^{\prime} \cup \mathscr{A}_{Z}^{\prime}$. Similarly, let $\mathscr{B}=\left\{B_{1}, B_{2}, \ldots, B_{|T|}\right\}$ and $\mathscr{B}^{\prime}=$ $\left\{B_{1}^{\prime}, B_{2}^{\prime}, \ldots, B_{|T|}^{\prime}\right\}$. We define also $\mathscr{D}$ to be a collection of $|T|+n-4 \beta(T)$ dummy rectangles, each of the size $\left(\frac{3}{4}-10 \delta, 1\right)$.

The collection of rectangles $\mathscr{A} \cup \mathscr{A}^{\prime} \cup \mathscr{B} \cup \mathscr{B}^{\prime} \cup \mathscr{D}$, together with a unit square bin is now viewed as an instance of the $2-\mathrm{BP}^{r}$ problem and denoted by $f(T)$. Our aim is to relate the optimum value $\mathrm{OPT}^{\prime}(f(T))$ of $2-\mathrm{BP}^{r}$ for an instance $f(T)$ to $\mathrm{OPT}(T)$. Informally, the dimensions of rectangles and dummy rectangles are chosen such that if $\mathrm{OPT}(T) \geq \beta(T)$, the rectangles can be packed into bins in such a way that their number is within a factor $(1+O(\delta))$ of the total area of rectangles. On the other hand, if $\operatorname{OPT}(T)<\frac{\beta(T)}{\gamma}$ for a constant $\gamma>1$, then the number of bins needed to pack all rectangles of $f(T)$ is larger than the total area of rectangles by a constant factor $\gamma^{\prime}>1$ independent of $\delta$ for $\delta>0$ small enough.

The reduction given by Bansal \& Sviridenko ([4]) can be viewed as a particular case of the Bin Packing reduction with $\delta=\frac{1}{500}$, a set $\mathcal{T}$ of instances $T \subseteq X \times Y \times Z$ of 3-bounded MAX-3DM with $|X|=|Y|=|Z|=q$, and a gap location $\beta(T)=q$ (as it follows from the Petrank's result [20]). The parameter $p$ is an important novelty of this paper. The crucial point is that for the proper choice of the parameter $p$ we can prove that even if rotations are allowed it is not advantageous to use them.

Remark 1. Bansal \& Sviridenko [4] (see also [3]) claim to prove not only nonexistence of APTAS (unless $\mathrm{P}=\mathrm{NP}$ ) for 2-DIMENSIONAL BIN PACKInG (without rotations), but also APX-hardness for it. However, such result does not follow from their proof. The given reduction from 3-bounded MAX-3DM to 2-BP is not an $L$-reduction (or an approximation preserving reduction), but it is rather a gap preserving reduction that preserves one but not all gaps. 
We start with the following simple lemma valid for the choice of $p \in\left[\frac{1}{4}+\right.$ $\left.9 \delta, \frac{1}{2}-20 \delta\right]$.

Lemma 1. (i) For every $r$-packing of $f(T)$ all rectangles from $\mathscr{A} \cup \mathscr{B}$ contained in the same bin are either in their initial orientations or all are rotated by ninety degrees.

(ii) For every r-packing of $f(T)$ if a bin contains exactly 4 rectangles from $\mathscr{A} \cup \mathscr{B}$, then all rectangles from $\mathscr{A} \cup \mathscr{B} \cup \mathscr{A}^{\prime} \cup \mathscr{B}^{\prime}$ packed in this bin are either in their initial orientations or all are rotated by ninety degrees.

For oriented packings some properties of the Bansal's and Sviridenko's reduction [4] (that corresponds to $p=0$ ) are preserved to our general situation with the parameter $p$ introduced. The proofs of Lemmas 3 and 4 given in [4] work in this case as well, as widths of rectangles are the same in both reductions.

Definition 1. ([4]) We say that two rectangles $A$ and $A^{\prime}$ from $\mathscr{A} \cup \mathscr{A}^{\prime} \cup \mathscr{B} \cup \mathscr{B}{ }^{\prime}$ are buddies if $\left\{A, A^{\prime}\right\}$ corresponds to a pair of rectangles for a single element from $X, Y, Z$ or $T$, e.g., $\left\{A, A^{\prime}\right\}=\left\{A_{X, i}, A_{X, i}^{\prime}\right\}$ for some $x_{i} \in X$ and similarly for the other sets $Y, Z$, and $T$.

Observation 1 For any rectangle, $A \in \mathscr{A}$ implies $w(A)+h(A)=\frac{3}{4}+p, A^{\prime} \in \mathscr{A}^{\prime}$ implies $w\left(A^{\prime}\right)+h\left(A^{\prime}\right)=\frac{3}{4}-p, B \in \mathscr{B}$ implies $w(B)+h(B)=\frac{3}{4}+p+9 \delta$, and $B^{\prime} \in \mathscr{B}^{\prime}$ implies $w\left(B^{\prime}\right)+h\left(B^{\prime}\right)=\frac{3}{4}-p-9 \delta$.

Observation 2 For any two rectangles $A, A^{\prime}$ in $\mathscr{A} \mathscr{A}^{\prime} \cup \mathscr{B} \cup \mathscr{B}^{\prime}, h(A)+h\left(A^{\prime}\right)=$ 1 if and only if $A$ and $A^{\prime}$ are buddies.

In the following lemma we observe some basic properties for oriented packing of rectangles from $\mathscr{A} \cup \mathscr{B} \cup \mathscr{A}^{\prime} \cup \mathscr{B}^{\prime}$ into unit square bin.

Lemma 2. Consider a unit square bin containing exactly 4 rectangles from $\mathscr{A} \cup$ $\mathscr{B}$ for an oriented packing of $f(T)$. Then the bin contains at most 8 rectangles from $\mathscr{A} \cup \mathscr{B} \cup \mathscr{A}^{\prime} \cup \mathscr{B}^{\prime}$ and if it contains exactly 8 rectangles then, for any $h \in\left[4 \delta, \frac{1}{2}-p-4 \delta\right]$, each rectangle intersects exactly one of the lines $L_{1}=$ $\{(x, y): y=h\}$ and $L_{2}=\{(x, y): y=1-h\}$.

Lemma 3. For any rectangles $A_{1}, A_{2}, A_{3} \in \mathscr{A}$ and $B \in \mathscr{B}, w\left(A_{1}\right)+w\left(A_{2}\right)+$ $w\left(A_{3}\right)+w(B)=1$ if and only if $\left\{A_{1}, A_{2}, A_{3}, B\right\}=\left\{A_{X, i}, A_{Y, j}, A_{Z, k}, B_{l}\right\}$ for some integers $i, j, k$, and $l$ such that $t_{l}=\left(x_{i}, y_{j}, z_{k}\right) \in T$. A similar statement holds also for rectangles $A_{1}^{\prime}, A_{2}^{\prime}, A_{3}^{\prime} \in \mathscr{A}^{\prime}, B^{\prime} \in \mathscr{B}^{\prime}$.

Lemma 4. Let $A_{1}, A_{2}, A_{3}, A_{4} \in \mathscr{A} \cup \mathscr{A}^{\prime}$ be such that no two of them are buddies. Then $\sum_{i=1}^{4} w\left(A_{i}\right) \neq 1$.

Definition 2. Given an r-packing of a bin by some rectangles from $f(T)$. The bin is called well-packed, if it contains exactly 4 rectangles from $\mathscr{A} \cup \mathscr{B}$ and 4 rectangles from $\mathscr{A}^{\prime} \cup \mathscr{B}^{\prime}$. 
Now the crucial fact is, that for any choice of the parameter $p$ from the interval $\left[\frac{1}{4}+9 \delta, \frac{1}{2}-20 \delta\right]$, we can characterize the structure of well-packed bins similarly as it has been done in [4] for oriented packings.

Lemma 5. A bin is well-packed if and only if it contains the rectangles $A_{X, i}$, $A_{Y, j}, A_{Z, k}, B_{l}, A_{X, i}^{\prime}, A_{Y, j}^{\prime}, A_{Z, k}^{\prime}, B_{l}^{\prime}$, for some $t_{l}=\left(x_{i}, y_{j}, z_{k}\right) \in T$.

Proof. The 8-tuple of rectangles corresponding to a triple as above can be packed in a square bin $\mathbb{B}=[0,1]^{2}$ even without using rotations. Starting from the bottom left corner of the bin $\mathbb{B}$ and moving to the right, each of rectangles $A_{X, i}$, $A_{Y, j}, A_{Z, k}$, and $B_{l}$ is placed such that it touches the bottom of the bin $\mathbb{B}$. As $w\left(A_{X, i}\right)+w\left(A_{Y, j}\right)+w\left(A_{Z, k}\right)+w\left(B_{l}\right)=1$ (Lemma 3$)$, the rectangles can be packed in this way. The rectangles $A_{X, i}^{\prime}, A_{Y, j}^{\prime}, A_{Z, k}^{\prime}$, and $B_{l}^{\prime}$ can be placed in the remaining gaps starting from the top left corner of the bin $\mathbb{B}$ and moving towards the right touching the top of the bin. Clearly, such packing is possible due to the size properties of rectangles.

Now we show that any well-packed bin contains rectangles that correspond to a triple in $T$. Due to Lemma 1(ii), all rectangles are either in their initial orientations or all are rotated by ninety degrees. We can assume that they are all in the initial orientation in a well-packed bin; the case when all are rotated by $90^{\circ}$ can be discussed similarly. Fix $h \in\left[4 \delta, \frac{1}{2}-p-4 \delta\right]$ and consider the lines $L_{1}=\{(x, y): y=h\}$ and $L_{2}=\{(x, y): y=1-h\}$. Due to Lemma 2, each rectangle must intersect exactly one of the lines $L_{1}$ and $L_{2}$. Moreover, as any rectangle has width larger than $\frac{1}{5}$, each of lines $L_{1}$ and $L_{2}$ intersects exactly 4 rectangles. Let $\left\{A_{1}, A_{2}, A_{3}, A_{4}\right\}$ denote the rectangles that intersect $L_{1}$ such that $A_{i}$ is to the left of $A_{j}$ for $i<j$. Similarly, let $\left\{A_{5}, A_{6}, A_{7}, A_{8}\right\}$ denote the rectangles that intersect $L_{2}$ in the left to right order. Thus, we have that

$$
\begin{gathered}
\sum_{i=1}^{4} w\left(A_{i}\right) \leq 1, \\
\sum_{i=1}^{4} w\left(A_{i+4}\right) \leq 1 .
\end{gathered}
$$

Observe that for each $i=1,2,3,4$ the rectangle $A_{i}$ must overlap with $A_{i+4}$ in the $x$-coordinate. Thus, we have that

$$
h\left(A_{i}\right)+h\left(A_{i+4}\right) \leq 1 \quad \text { for } i=1,2,3,4 .
$$

From (3) it follows that, for each $i=1,2,3,4$, at most one of $A_{i}, A_{i+4}$ belongs to $\mathscr{A} \cup \mathscr{B}$. Consequently, for each $i=1,2,3,4$ exactly one of $A_{i}, A_{i+4}$ is from $\mathscr{A} \cup \mathscr{B}$ and another one is from $\mathscr{A}^{\prime} \cup \mathscr{B}^{\prime}$. Using these facts, we can use the same arguments as in [4]:

(i) First observe that at most 1 from rectangles $\left\{A_{1}, \ldots, A_{8}\right\}$ belongs to $\mathscr{B}$. Indeed, if $k \geq 2$ of them belong to $\mathscr{B}$ and $4-k$ belong to $\mathscr{A}$, then the sum of widths of these rectangles from $\mathscr{A} \mathscr{B}$ would be $>1$, a contradiction with the fact that any line in $y$-direction intersects at most 1 rectangle from $\mathscr{A} \cup \mathscr{B}$. 
(ii) If no rectangle from $\left\{A_{1}, \ldots, A_{8}\right\}$ belongs to $\mathscr{B}$, than the same is true for $\mathscr{B}^{\prime}$. The height of any rectangle in $\mathscr{B}^{\prime}$ is larger then $\frac{1}{2}-p-\delta$ so such a rectangle cannot form a pair $\left\{A_{i}, A_{i+4}\right\}$ with a rectangle from $\mathscr{A}$. Thus, in this case four rectangles belong to $\mathscr{A}$ and four to $\mathscr{A}^{\prime}$. Using Observation 1 we get $\sum_{i=1}^{8}\left(w\left(A_{i}\right)+h\left(A_{i}\right)\right)=6$, thus it must be the case that each of (1), (2) and (3) must hold with equality. By Observation 2, $A_{i}$ and $A_{i+4}$ are buddies for each $i=1,2,3,4$. In particular, no two rectangles among $A_{1}, A_{2}, A_{3}$, and $A_{4}$ are buddies. Now Lemma 4 contradicts with $\sum_{i=1}^{4} w\left(A_{i}\right)=1$ that has been observed earlier. Thus this case is impossible.

So, necessarily exactly one of rectangles $\left\{A_{1}, A_{2}, \ldots, A_{8}\right\}$ belongs to $\mathscr{B}$, say $B_{l}$.

(iii) As, due to (3), no pair $\left\{A_{i}, A_{i+4}\right\}$ can contain a rectangle from $\mathscr{B}^{\prime}$ and a rectangle from $\mathscr{A}$, there can be at most one rectangle from $\mathscr{B}^{\prime}$. But if there are no rectangles from $\mathscr{B}^{\prime}$, then the sum of widths of all 8 rectangles would be $>2$, a contradiction.

Consequently, there is exactly 1 rectangle from $\mathscr{B}^{\prime}, 1$ from $\mathscr{B}, 3$ from $\mathscr{A}$, and 3 from $\mathscr{A}^{\prime}$. Using Observation 1 we get $\sum_{i=1}^{8}\left(w\left(A_{i}\right)+h\left(A_{i}\right)\right)=6$, thus each of (1), (2), and (3) holds with equality. In particular, for each $i=1,2,3,4, A_{i}$ and $A_{i+4}$ are buddies due to Observation 2. Let $m \in\{1,2\}$ be such that $B_{l}$ intersects the line $L_{m}$. Let $A_{m_{1}}, A_{m_{2}}, A_{m_{3}}$ denote the other three rectangles (from $\mathscr{A} \cup \mathscr{A}^{\prime}$ ) which are also intersected by $L_{m}$. Thus we have that $w\left(A_{m_{1}}\right)+$ $w\left(A_{m_{2}}\right)+w\left(A_{m_{3}}\right)+w\left(B_{l}\right)=1$. None of $A_{m_{1}}, A_{m_{2}}, A_{m_{3}}$ can lie in $\mathscr{A}^{\prime}$ because otherwise $w\left(A_{m_{1}}\right)+w\left(A_{m_{2}}\right)+w\left(A_{m_{3}}\right)+w\left(B_{l}\right)>\left(\frac{1}{4}+8 \delta\right)+\left(\frac{1}{4}+\delta\right)+2\left(\frac{1}{4}-4 \delta\right)=$ $1+\delta$, a contradiction. Hence $\left\{A_{m_{1}}, A_{m_{2}}, A_{m_{3}}\right\} \subseteq \mathscr{A}$, and using Lemma 3 we get that $\left\{A_{m_{1}}, A_{m_{2}}, A_{m_{3}}\right\}=\left\{A_{X, i}, A_{Y, j}, A_{Z, k}\right\}$ for integers $i, j, k$ such that $t_{l}=\left(x_{i}, y_{j}, z_{k}\right)$, where $t_{l}$ is the corresponding triple for the rectangle $B_{l}$. This completes the proof.

Now we can prove the main theorem of this section

Theorem 1. There is a constant $\rho>1$ such that it is NP-hard to approximate 2-Dimensional Bin PACKing With Rotations into unit square bins with an asymptotic approximation ratio less than $\rho$.

Proof. Recall that the Bin Packing reduction $f$ started from a set $\mathcal{T}$ of instances of MAX-3DM such that for $T \in \mathcal{T}$ it is NP-hard to decide of whether $\operatorname{OPT}(T) \geq$ $\beta(T)$, or $\operatorname{OPT}(T)<\alpha(T)$.

(a) Assume first that $T \in \mathcal{T}$ is such that $\mathrm{OPT}(T) \geq \beta(T)$. We will show that the corresponding instance $f(T)$ of the $2-\mathrm{BP}^{r}$ problem has its optimum $\mathrm{OPT}^{\prime}(f(T))$ of size at most $|T|+n-3 \beta(T)$. Consider a matching $M$ in $T$ consisting of $\beta(T)$ triples. For each triple $t_{l}=\left(x_{i}, y_{j}, z_{k}\right) \in M$ we create a wellpacked bin with rectangles $\left\{A_{X, i}, A_{Y, j}, A_{Z, k}, B_{l}, A_{X, i}^{\prime}, A_{Y, j}^{\prime}, A_{Z, k}^{\prime}, B_{l}^{\prime}\right\}$ packed.

For each $t_{l} \in T \backslash M$ we can put $B_{l}$ and $B_{l}^{\prime}$ along with a dummy rectangle into a bin; in this way we use $|T|-\beta(T)$ dummy rectangles.

For each of $n-3 \beta(T)$ elements in $X \cup Y \cup Z$ that are not covered by $M$, we put in a bin the corresponding buddies $A$ and $A^{\prime}$ along with one dummy rectangle. The rest of the dummy rectangles is used in this way and all rectangles from $f(T)$ are packed into $|T|+n-3 \beta(T)$ bins. 
(b) Assume now that $T \in \mathcal{T}$ satisfies $\operatorname{OPT}(T)<\alpha(T)$. Our aim is to estimate $\mathrm{OPT}^{\prime}(f(T))$ from below. Consider for an instance $f(T)$ any feasible solution of $2-\mathrm{BP}^{r}$. There will be exactly $N_{d}=|T|+n-4 \beta(T)$ bins with dummy rectangles, each of them can contain at most one rectangle from $\mathscr{A} \cup \mathscr{B}$. Let us consider now bins without dummy rectangles. If such bin is not well-packed then it either contains at most 3 rectangles from $\mathscr{A} \cup \mathscr{B}$ or else it contains at most 3 rectangles from $\mathscr{A}^{\prime} \cup \mathscr{B}^{\prime}$. Let $N_{g}$ denote the number of well-packed bins. Among the bins without dummy rectangles which are not well-packed, let $N_{b_{2}}$ denote the number of bins with at most 3 rectangles from $\mathscr{A} \cup \mathscr{B}$, and let $N_{b_{1}}$ denote the number of the rest rectangles (i.e., $N_{b_{1}}$ is the number of bins with 4 rectangles from $\mathscr{A} \backsim \mathscr{B}$, but with at most 3 rectangles from $\left.\mathscr{A}^{\prime} \cup \mathscr{B}^{\prime}\right)$.

Since all $|T|+n$ rectangles from $\mathscr{A} \cup \mathscr{B}$ have to be packed, we have the constraint that

$$
4 N_{g}+4 N_{b_{1}}+3 N_{b_{2}}+N_{d} \geq|T|+n,
$$

or equivalently

$$
4 N_{g}+4 N_{b_{1}}+3 N_{b_{2}} \geq 4 \beta(T) .
$$

With the choice of parameter $p=\frac{1}{4}+9 \delta$ and assuming $\delta \in\left(0, \frac{1}{500}\right]$ as small as we need, rectangles from $\mathscr{A} \cup \mathscr{B}$ are roughly $\left(\frac{1}{4}, \frac{3}{4}\right)$ each, and those from $\mathscr{A}^{\prime} \cup \mathscr{B}^{\prime}$ are roughly $\left(\frac{1}{4}, \frac{1}{4}\right)$ each. In what follows we will count rectangles from $\mathscr{A} \cup \mathscr{B}$ with weight 3 , and those from $\mathscr{A}^{\prime} \cup \mathscr{B}^{\prime}$ with weight 1 each. Easy area's estimate shows that the total weight of rectangles packed to a unit square bin cannot exceed 16. Further, any bin containing a dummy rectangle can contain rectangles from $\mathscr{A} \cup \mathscr{B} \cup \mathscr{A}^{\prime} \cup \mathscr{B}^{\prime}$ of weight at most 4 . Observe that each of $N_{b_{1}}$ bins contains rectangles of weight at most 15 . Hence the second constraint derived from the fact that all rectangles have to be packed reads as follows:

$$
16 N_{g}+15 N_{b_{1}}+16 N_{b_{2}}+4 N_{d} \geq 4(|T|+n) .
$$

Using $N_{d}=|T|+n-4 \beta(T)$ and adding the constraint (4) to the last one we get

$$
20 N_{g}+19 N_{b_{1}}+19 N_{b_{2}} \geq 20 \beta(T) .
$$

Since the set of well-packed bins corresponds to a feasible solution for a matching (by Lemma 5 ), $N_{g}<\alpha(T)$. Thus, assuming $\mathrm{OPT}(T)<\alpha(T)$ we get

$$
\begin{aligned}
\operatorname{OPT}^{\prime}(f(T)) & >N_{g}+N_{b_{1}}+N_{b_{2}}+N_{d} \geq \frac{20}{19} \beta(T)-\frac{1}{19} N_{g}+N_{d} \\
& >|T|+n-3 \beta(T)+\frac{1}{19}(\beta(T)-\alpha(T)) .
\end{aligned}
$$

It easily follows that our reduction $f$ is a gap preserving reduction assuming that we started from $(\alpha(T), \beta(T))$-gap version of the bounded MAX-3DM problem for which $\frac{\beta(T)-\alpha(T)}{|T|+n-3 \beta(T)}$ is bounded below by a positive constant.

Now suppose that for a fixed constant $\rho, 1<\rho<1+\frac{1}{19} \frac{\beta(T)-\alpha(T)}{|T|+n-3 \beta(T)}$, there exists a polynomial time algorithm $\mathcal{A}_{\rho}$ and a constant $C$ such that for instances $f(T)$ if $\mathrm{OPT}^{\prime}(f(T))>C$, then $\mathcal{A}_{\rho} \leq \rho \mathrm{OPT}^{\prime}(f(T))$. Thus, for any corresponding 
instance $T$ of MAX-3DM we could distinguish whether $\mathrm{OPT}(T) \geq \beta(T)$, or $\operatorname{OPT}(T)<\alpha(T)$, which is an NP-hard problem. Hence, it is NP-hard to achieve an asymptotic approximation ratio $\leq \rho$ for the problem 2-DIMENSIONAL BIN PaCking With Rotations into unit square bins.

Using the NP-hard gap result from Theorem A we can obtain an explicit lower bound $1+\frac{1}{3792}$ on asymptotic approximation ratio of any polynomial time approximation algorithm (unless $\mathrm{P}=\mathrm{NP}$ ) for 2-Dimensional Bin PACKING With Rotations into unit square bins. For the same problem without rotations our method provides a lower bound $1+\frac{1}{2196}$.

\subsection{3-dimensional Strip Packing problems}

Let a list of 2-dimensional rectangles $\mathscr{L}=\left\{\left(r_{1}^{1}, r_{2}^{1}\right),\left(r_{1}^{2}, r_{2}^{2}\right), \ldots,\left(r_{1}^{n}, r_{2}^{n}\right)\right\}$ with a bin $\mathbb{B}=\left(b_{1}, b_{2}\right)$ be an instance of the 2-Dimensional Bin PACKInG problem. For a fixed $t>0$ we define an instance of the 3 -Dimensional Strip PaCking problem as a list of 3-dimensional rectangles $\mathscr{L}_{t}=\left\{\left(r_{1}^{i}, r_{2}^{i}, t\right): 1 \leq i \leq n\right\}$ with a strip $\left(b_{1}, b_{2}, \infty\right)$. It is easy to prove, that if $\mathrm{OPT}(\mathscr{L})$ denote the optimum of an instance $\mathscr{L}$ for 2 -BP (resp., $2-\mathrm{BP}^{r}$ ) then $t \cdot \mathrm{OPT}(\mathscr{L})$ denote the optimum of the corresponding 3-dimensional instance $\mathscr{L}_{t}$ for 3 -SP (resp., for 3 -SP ${ }^{z}$ and 3 -SP provided $\left.t>\max \left\{b_{1}, b_{2}\right\}\right)$.

Hence, non-existence of APTAS for 2-BP ([4]) implies non-existence of APTAS for the 3-SP problem, unless $\mathrm{P}=\mathrm{NP}$. Moreover, using a heterogeneous scaling one can obtain some inapproximability results also for $3-\mathrm{SP}^{z}$ and $3-\mathrm{SP}^{r}$ already from hardness results for 2-BP, e.g., for instances of $3-\mathrm{SP}^{z}$ and $3-\mathrm{SP}^{r}$ with a strip $(b, 1, \infty)$ for any fixed $b \in\left(0, \frac{1}{2}\right)$. However, for a strip with square base we have to use Theorem 1 instead.

Theorem 2. There is no APTAS for any of 3-dimensional strip packing problems $3-\mathrm{SP}, 3-\mathrm{SP}^{z}$, and $3-\mathrm{SP}^{r}$ on instances with a strip $(1,1, \infty)$, unless $\mathrm{P}=\mathrm{NP}$.

\section{Maximum Rectangle Packing Problem}

Another rectangle bin packing problem well studied in the literature (e.g., [13], $[2])$ is the following:

Definition 3. Given a collection of d-dimensional rectangles together with a $d$-dimensional rectangular bin $\mathbb{B}$. The goal of the MAXIMUM $d$-DIMENSIONAL RECTANGLE PACKING problem is to pack the maximum number of rectangles from the collection into a single bin $\mathbb{B}$.

Other variants of this problem are studied as well, e.g., each of the rectangles can be associated with weight, and the goal is to maximize the total weight of packed rectangles. In some variants ninety-degree rotations of rectangles are allowed. But even in the simplest case, namely the 2-dimensional unweighted case without rotations, only a $(2+\varepsilon)$-approximation algorithm is known [13]. The question of whether there is an APTAS is open. However, in the 3-dimensional case the problem can be settled in the negative. 
Theorem 3. Unless $\mathrm{P}=\mathrm{NP}$, there is no APTAS for the MAXIMUM 3-DIMENSIONAL RECTANGLe PACKIng problem with unit cube bin. The same result holds also for $z$-oriented packings and for r-packings, in both cases with a bin $(1,1, b)$, where $b \in\left(0, \frac{1}{4}\right)$.

Proof. We can use the hardness result of Theorem 2 for 3-SP with the strip $(1,1, \infty)$. Namely, there is a constant $\rho>1$ and an infinite family $\mathcal{F}$ of instances of the 3 -SP problem with the strip $(1,1, \infty)$, such that for a certain computable function $\alpha: \mathcal{F} \rightarrow \mathbb{N}$ it is NP-hard to distinguish for $\mathscr{L} \in \mathcal{F}$ whether $\operatorname{OPT}(\mathscr{L}) \leq \alpha(\mathscr{L})$, or $\operatorname{OPT}(\mathscr{L})>\rho \cdot \alpha(\mathscr{L})$. Moreover, each rectangle in $\mathscr{L}$ is a small perturbation of either $\left(\frac{1}{4}, \frac{1}{2}, 1\right)$ or $\left(\frac{3}{4}, 1,1\right)$.

For oriented packings (i.e., without rotations) and for any $\mathscr{L} \in \mathcal{F}$ denote by $\mathscr{L}^{\prime}$ a rescaled copy of $\mathscr{L}$ by a factor $1 / \alpha(\mathscr{L})$ in the direction of the $z$-axis. Then clearly, it is NP-hard to decide whether $\mathrm{OPT}\left(\mathscr{L}^{\prime}\right) \leq 1$, or $\mathrm{OPT}\left(\mathscr{L}^{\prime}\right)>\rho$ for an instance $\mathscr{L}$ of the 3 -SP problem with the strip $(1,1, \infty)$. In the former case all rectangles of $\mathscr{L}^{\prime}$ can be packed into the unit cube bin. In the latter one we easily obtain that less than $\left|\mathscr{L}^{\prime}\right|-\lfloor(\rho-1) \alpha(\mathscr{L})\rfloor$ can be packed into this bin.

For $z$-oriented packings we can use the same arguments starting instead from the NP-hard gap derived for the problem $2-\mathrm{BP}^{r}$ with unit square bin $[0,1]^{2}$ (the proof of Theorem 1).

For $r$-packings we rescale $\mathscr{L}$ by a factor $b / \alpha(\mathscr{L}), b \in\left(0, \frac{1}{4}\right)$, in the direction of $z$-axis. Then it is NP-hard to decide whether all, or only a fraction strictly less than 1 of the rectangles of $\mathscr{L}$ can be packed into the bin $(1,1, b)$. The special uniform structure of instances in our hardness result for 2- $\mathrm{BP}^{r}$ implies that all $r$-packings for such rescaled instances are, in fact, $z$-oriented packings. Thus the results follow as above.

\section{References}

1. G. Ausiello, P. Crescenzi, G. Gambosi, V. Kann, A. Marchetti-Spaccamela, and M. Protasi, Complexity and approximation, Springer, 1999.

2. B. S. Baker, A. R. Calderbank, E. G. Coffman, and J. C. Lagarias, Approximation algorithms for maximizing the number of squares packed into a rectangle, SIAM J. on Algebraic and Discrete Methods 4 (1983), 383-397.

3. N. Bansal, J. R. Correa, C. Kenyon, and M. Sviridenko, Bin packing in multiple dimensions: inapproximability results and approximation schemes, Manuscript, February 2004.

4. N. Bansal and M. Sviridenko, New approximability and inapproximability results for 2-dimensional bin packing, Proceedings of the 15th Annual ACM-SIAM Symposium on Discrete Algorithms, SODA, 2004, pp. 189-196.

5. A. Caprara, Packing 2-dimensional bins in harmony, Proceedings of the 43th Annual IEEE Symposium on Foundations of Computer Science, FOCS, 2002, pp. 490499.

6. C. Chekuri and S. Khanna, On multi-dimensional packing problems, Proc. of the 10th ACM-SIAM Symposium on Discrete Algorithms, SODA, 1999, pp. 185-194.

7. M. Chlebík and J. Chlebíková, Complexity of approximating bounded variants of optimization problems, Theoretical Computer Science, (to appear), DOI: $10.1016 /$ j.tcs.2005.11.029. 
8. J. R. Correa and C. Kenyon, Approximation schemes for multidimensional packing, Proceedings of the 15th ACM-SIAM Symposium on Discrete Algorithms, SODA, 2004, pp. 179-188.

9. J. Csirik and A. van Vliet, An on-line algorithm for multidimensional bin packing, Operation Research Letters 13 (1993), 149-158.

10. W. Fernandez de la Vega and G. S. Lueker, Bin packing can be solved within $(1+\varepsilon)$ in linear time, Combinatorica 1 (1981), 349-355.

11. K. Jansen and R. Solis-Oba, An asymptotic approximation algorithm for $3 d$-strip packing, to appear in Proceedings of the 17th Annual ACM-SIAM Symposium on Discrete Algorithms, SODA, 2006.

12. K. Jansen and R. Stee, On strip packing with rotations, Proceedings of the 37th ACM Symposium on Theory of Computing, STOC, 2005, pp. 755-761.

13. K. Jansen and G. Zhang, On rectangle packing: maximizing benefits, Proceedings of the 15th Annual ACM-SIAM Symposium on Discrete Algorithms, SODA, 2004, pp. 197-206.

14. V. Kann, Maximum bounded 3-dimensional matching is MAX SNP complete, Information Processing Letters 37 (1991), 27-35.

15. N. Karmarkar and R. M. Karp, An efficient approximation scheme for the onedimensional bin-packing problem, Proceedings of the 23rd IEEE Symposium on Foundations of Computer Science, FOCS, 1982, pp. 312-320.

16. C. Kenyon and E. Rémila, A near optimal solution to a two-dimensional cutting stock problem, Mathematics of Operations Research 25 (2000), 645-656, Preliminary version in Proceedings of the 37 th Annual IEEE Symposium on Foundations of Computer Science, FOCS, 1996, pp. 31-36.

17. K. Li and K. H. Cheng, On three-dimensional packing, SIAM J. Comput. 19 (1990), 847-867.

18. F. K. Miyazawa and Y. Wakabayashi, Approximation algorithms for the orthogonal z-oriented three-dimensional packing problems, SIAM J. Comput. 29 (2000), 10081029.

19. , Packing problems with orthogonal rotations, Proceedings of the 6th Latin American Symposium on Theoretical Informatics, LATIN, Buenos Aires, Argentina, LNCS 2976, Springer-Verlag, 2004, pp. 359-368.

20. E. Petrank, The hardness of approximation: Gap location, Computational Complexity 4 (1994), 133-157.

21. G. J. Woeginger, There is no asymptotic PTAS for two-dimensional vector packing, Information Processing Letters 64(6) (1997), 293-297. 